\title{
Some Relations Between Dimension and Lyapounov Exponents
}

\author{
F. Ledrappier \\ Laboratoire de Probabilités, 9, Quai St. Bernard-T.56, F-752 30 Paris, Cedex 05, France
}

\begin{abstract}
We consider differentiable maps and compact invariant sets. We introduce dimensional quantities related to the ergodic invariant measures, and prove some simple relations.
\end{abstract}

We consider differentiable maps and compact invariant sets. An estimate from above for the Hausdorff dimension of such a set has been given by A. Douady and J. Oesterlé $[D O]$ and by Mañe $\left[M_{1}\right]$. In this paper we discuss some other relations of this kind. We first show how to deduce an estimate involving Lyapunov exponents of the system. We also introduce the fractal dimension $f(m)$ of a measure $m$ on a compact space, which weights an "essential" dimension of $(X, m)$.

The results are the following: for any ergodic invariant probability measure, we consider the spectrum of the linear tangent map (the so-called Lyapunov exponents) and the "dilating dimension" of this spectrum dim dil Sp $m$; the dimension of a compact invariant set is bounded from above by the supremum of dim dil Sp $m$ over all invariant probability measures; individually, for any ergodic invariant probability measure, we have

$$
f(m) \leqq \operatorname{dim} \operatorname{dil} \operatorname{Sp} m .
$$

This inequality is generally a strict inequality, as is shown by considering maps of the interval, where $f(m)$ is related to the entropy $h(m)$ and the positive Lyapunov coefficient $\lambda$ by $f(m)=\frac{h(m)}{\lambda}$.

This notion of dimension of a measure is closer to what is actually measured in experiments like those performed by P. Frederikson, J. Kaplan and J. Yorke [FKY]. It leads us to reformulate these conjectures there and to discuss some other questions.

\section{Notations and Results}

Let $L$ be a linear operator from an euclidean space $E$ of dimension $d$ in an euclidean space $F$. Define $s$-numbers of $L$, denoted $[L]$, as the decreasing sequence 
$\lambda_{1} \geqq \lambda_{2} \ldots \geqq \lambda_{d}$ of logarithms of the eigenvalues of the positive operator $\left(L^{*} L\right)^{1 / 2}$ (multiple eigenvalues are repeated according to their multiplicity).

For any such sequence $s=\lambda_{1}, \ldots, \lambda_{d}$, and any real $\alpha, 0 \leqq \alpha \leqq d$, we define $c^{\alpha}(s)$ by $c^{\alpha}(s)=\sum_{i=1}^{[\alpha]} \lambda_{i}+(\alpha-[\alpha]) \lambda_{[\alpha]+1}$, where $[\alpha]$ denotes the integer part of the number $\alpha$, and dim dil $s$ by

$$
\begin{aligned}
& \operatorname{dim} \operatorname{dil} s=0 \text { if } \lambda_{1}<0, \\
& \operatorname{dim} \operatorname{dil} s=\sup \left\{\alpha, 0 \leqq \alpha \leqq d, c^{\alpha}(s) \geqq 0\right\} \text { otherwise. } \\
& \text { (We can put } \left.c^{\alpha}(s)=-\infty \text { for } \alpha>d\right) .
\end{aligned}
$$

Let $K$ be a compact set in a metric space. For any $\beta>0$, any $\varepsilon>0$, and any cover $u$ of $K$ by sets $A_{i}$ of diameter $r_{i}, i \in I, r_{i} \leqq \varepsilon$, we compute $N_{\beta}(u, \varepsilon)=\sum_{i \in I} r_{i}^{\beta}$ and

$$
M_{\beta}(K)=\liminf _{\varepsilon \downarrow 0} N_{\beta}(u, \varepsilon) .
$$

The Hausdorff dimension of $K \operatorname{dim} K$ is defined by

$$
\operatorname{dim} K=\inf \left\{\beta \beta \geqq 0 \mid M_{\beta}(K)=0\right\} .
$$

If $f$ is a differentiable map of Riemannian manifold $X$, and $K$ a compact invariant set, we have by Douady and Oesterle's formula

$$
\operatorname{dim} K \leqq \sup _{x}\left\{\operatorname{dim} \operatorname{dil}\left[D_{x} f\right]\right\} .
$$

For any measure $m$ on a compact metric space $(K, d)$, for any $\varepsilon>0$ and $\delta>0$, we call $N_{d}(\varepsilon, \delta, m)$ the smallest number of balls of radius $\varepsilon$ we need to cover the space up to measure $\delta$. We then define the dimension of $m$ by

$$
f(m)=\lim _{\delta \rightarrow 0} \lim \sup \frac{\log N_{d}(\varepsilon, \delta, m)}{\log 1 / \varepsilon} .
$$

For an invariant ergodic measure $m$ by a $\operatorname{map} f$, we shall use a definition of the entropy very close to the preceding one (cf. Katok $[\mathrm{K}]$ ): let $d_{n}$ be the metric on $K$ defined by

$$
d_{n}(x, y)=\max \left\{d\left(f^{i} x, f^{i} y\right), 0 \leqq i \leqq n\right\} .
$$

We define the entropy $h(m)$ by

$$
h(m)=\lim _{\varepsilon \downarrow 0} \lim _{\delta \downarrow 0} \lim \sup \frac{1}{n} \log N_{d_{n}}(\varepsilon, \delta, m) .
$$

Let $P=\left\{p_{1}, p_{2}, \ldots, p_{n}, \ldots\right\}$ be a partition of $X$ into measurable sets and define $r(x)$ by $x \in p_{r}(x)$. If $H(P)=-\int \log m\left(P_{r(x)}\right) m(d x)<+\infty$, we have, by Shannon-Mac Millan's theorem (cf [B])

$$
\varlimsup_{n}-\frac{1}{n} \log m\left(p_{r}(x) \cap f^{-1} p_{r(f x) \cap \cdots \cap} f^{-n+1} p_{r\left(f^{n-1} x\right)}\right) \leqq h(m) .
$$

If we consider a differentiable map $f$ of a compact Riemannian manifold $X$, 
we denote $\mathscr{E}(x, f)$ the set of invariant ergodic probability measures. For any $m$ in $\mathscr{E}(x, f)$, there exists a sequence $\mathrm{Sp} m$ of $s$ numbers such that

$$
\frac{1}{n}\left[D_{x} f^{n}\right] \rightarrow \operatorname{Sp} m \text { a.e.and in } L^{1} .
$$

Furthermore by Oseledets' theorem (cf. [O], $[\overline{\mathrm{R}}],[\overline{\mathrm{R}} \mathrm{a}]$ ), there exists almost everywhere a decreasing family of subspaces of $T_{x} X$,

$$
T_{x} X=E_{s_{1}}^{x} \supset E_{s_{2}}^{x} \supset \ldots \supset E_{s_{r}}^{x} \supset E_{s_{r+1}}^{x}=\{0\}
$$

such that the map $x \rightarrow E_{s_{i}}^{x}$ is measurable and for almost all $x$, the sequence $\frac{1}{n} \log \left\|D_{x} f^{n} v\right\|$ converges uniformly on $v$ in $E_{s_{j}}^{x} \backslash E_{s_{j}+1}^{x}$ towards some element $\lambda_{s_{j}}$ of Sp $m$.

Our results are the following:

Proposition 1. If $f$ is a differentiable map of a compact Reimannian manifold $X$, and $m$ an invariant ergodic probability measure, $f(m) \geqq \frac{h(m)}{\lambda_{1}}$, where $\lambda_{1}$ is the first element of $\operatorname{Sp} m$. (Proposition 1 is empty when $\lambda_{1}=0$ ).

Proposition 2. If $f$ is a differentiable map of a Reimannian manifold $X$ and $K a$ invariant subset,

$$
\inf _{n} \sup _{x \in K} \operatorname{dim} \operatorname{dil}\left[D_{x} f^{n}\right]=\sup _{m \in \mathscr{E}(K, f)} \operatorname{dim} \operatorname{dil} \operatorname{Sp} m .
$$

Corollary. With the same conditions, we have

$$
\operatorname{dim} K \leqq \sup _{m \in \mathscr{E}(K, f)} \operatorname{dim} \operatorname{dil} \operatorname{Sp} m .
$$

Proposition 3. If $f$ is a differentiable map of a compact Riemannian manifold $X$ such that $D_{x}$ fis Hölder continuous on $X$ and if $m \in \mathscr{E}(X, f)$, we have

$$
f(m) \leqq \operatorname{dim} \operatorname{dil~Sp} m .
$$

Let us make some comments on these results.

Proposition 1 gives a rough estimate, but in certain cases it may be the best one; for instance, in dimension 1 , we have the following generalization of known results (cf. [B], [Bo], [C], [E], [F]).

Proposition 4. Let $f$ be a piecewise differentiable map from an interval I into itself, such that $f^{\prime}$ is piecewise monotone-Let $Q$ be the partition of I defined by the critical points of $f$ and of $f^{\prime}$ - Let $m$ be an invariant ergodic probability measure such that $H(Q)<+\infty$ and $\int \log \left|f^{\prime}\right| d m>0$. We have

$$
f(m)=\frac{h(m)}{\int \log \left|f^{\prime}\right| d m} .
$$

There are examples where the estimate in the corollary is an equality, for instance when an ergodic smooth measure exists, but there are also examples where this is not true (cf. the discussion in [FKY] and below). The result of Proposition 3 is 
also an equality for an ergodic smooth measure and in a forthcoming paper, it will be shown that we still have $f(m)=\operatorname{dim} \operatorname{dil~Sp} m$ if the following conditions are satisfied: there is only one nonstrictly positive exponent and the measure $m$ is absolutely continuous with respect to the unstable foliation. (L. S. Young has also obtained a related result.)

Let us also remark that all these results can be extended to a differentiable map of a Hilbert space satisfying suitable compactness conditions as long as ergodic theorems (cf. $\left[\mathrm{R}_{2}\right]$ ) and [D.O] are still valid. In the case of a differentiable map of a Banach space, Spectrum and Lyapunov are defined by an extension of the Oseledets theorem $\left[\mathrm{M}_{2}\right]$, and Mañe $\left(\left[\mathrm{M}_{1}\right]\right)$ gave a formula for the capacity of a compact invariant set very close to the one we get by corollary here. The capacity of a compact set $K$ is given by $c(K)=\lim _{\varepsilon} \sup \frac{\log N_{d}(\varepsilon, o, m)}{\log 1 / \varepsilon}$, where $m$ is any measure with support the whole set $K$. Although it is not explicitly stated that way, Mañe's estimation could actually give with the help of Proposition 2,

$$
c(K) \leqq \sup _{m \in \mathscr{E}(K, f)} \operatorname{dim} \operatorname{dil} \operatorname{Sp} m
$$

\section{Some Proofs}

We first prove Proposition 1. We put

Let $f$ be a differentiable map of a compact Riemannian manifold $X$, and $r>0$.

$$
\left\|D_{x} f\right\|_{r}=\max \left(\left\|D_{x} f\right\|, \frac{1}{r}\right) \text {. }
$$

For any $\chi>0$, there exists $\varepsilon>0$ such that $d(x, y)<\varepsilon$ implies $\left\|D_{y} f\right\| \leqq\left\|D_{x} f\right\|_{r}$ $(1+\chi)$. Let $m$ be an invariant ergodic measure, $\delta>0$ and let us denote $v_{r}(f)=$ $\int \log \left\|D_{x} f\right\|_{r} m(d x)$.

By the ergodic theorem, there exists $n_{0}$ such that if $n \geqq n_{0} m\left(A_{n}\right) \geqq 1-\delta$, where

$$
A_{n}=\left\{x ; \sup _{0 \leqq j \leqq n} \prod_{k=0}^{j-1}\left\|D_{f_{x}^{k}} f\right\|_{r} \leqq e^{n v_{r}(f)(1+\chi)}\right\}
$$

Let $\varepsilon^{\prime}<\varepsilon$, if $x \in A_{n}$ and $d(y, x) \leqq \varepsilon^{\prime} e^{-n v_{r}(f)(1+\chi)}(1+\chi)^{-n}$, we have clearly by induction on $j \leqq n$,

$$
d\left(f^{j} y, f^{i} x\right)<\varepsilon^{\prime} e^{-n v_{r}(f)(1+\chi)} \prod_{k=0}^{j-1}\left\|D_{f_{x}^{k}} f\right\|_{r}(1+\chi)^{j-n}
$$

In the other words the $d$ ball of radius $\varepsilon^{\prime} e^{-n v_{r}(f)(1+\chi)}(1+\chi)^{-n}$ and center $x$ is contained in the $d_{n}$ ball of radius $\varepsilon^{\prime}$. Furthermore, in a cover up to measure $\delta$ by a family of sets, the subcover made of the sets which meet $A_{n}$ by a nonempty intersection covers $X$ up to a measure $2 \delta$.

And putting this together we have just shown

$$
N_{d_{n}}\left(\varepsilon^{\prime}, 2 \delta, m\right) \leqq N_{d}\left(\frac{\varepsilon^{\prime} e^{-n v_{r}(f)(1+\chi)}(1+\chi)^{-n}}{2}, \delta, m\right) .
$$


By taking $\lim _{\varepsilon^{\prime} \rightarrow 0} \lim _{\delta \rightarrow 0} \lim _{n} \sup \frac{1}{n} \log$, we have, if $v_{r}(f)>0$

$$
h(m) \leqq f(m) \cdot\left[v_{r}(f)(1+\chi)+\log (1+\chi)\right] .
$$

But $\chi$ and $r$ can be arbitrarily small. Therefore we get $h(m) \leqq f(m)$. $\left(\int \log \left\|D_{x} f\right\| m(d x)\right)^{+}$and by applying this result to $f^{n} n \geqq 0$, we have finally $h(m) \leqq f(m) \cdot \frac{1}{n}\left(\int \log \left\|D_{x} f^{n}\right\| m(d x)\right)^{+}$which by (1.2) proves Proposition 1.

Before proving Proposition 2, we have the following Lemma.

Lemma 2.1. Let $L$ and $L^{\prime}$ be linear operators from respectively euclidean spaces $E$ to $E^{\prime}$ and $E^{\prime}$ to $E^{\prime \prime}$. We have for any real $\alpha$

$$
c^{\alpha}\left(\left[L^{\prime} . L\right]\right) \leqq c^{\alpha}([L])+c^{\alpha}\left(\left[L^{\prime}\right]\right) .
$$

Proof. It is clear that if one of $c^{\alpha}([L])$ or $c^{\alpha}\left(\left[L^{\prime}\right]\right)$ is $-\infty$, that means that either rank of $L$ or $L^{\prime}$ is smaller than $\alpha$, so that the rank of $L^{\prime} . L$ is also smaller than $\alpha$ and (2.1) is valid.

Remark also that $c^{1}([L])$ is the logarithm of the norm of the operator $L$ and that proves (2.1) for $\alpha=1$. The proof of (2.1) for an integer $\alpha$ follows by considering the wedge product $\stackrel{\alpha}{\wedge}$ Land applying $c^{\alpha}([L])=c^{1}([\stackrel{\alpha}{\wedge} L])$.

Then (2.1) follows for a rational $\alpha=\frac{p}{q}$ by considering the direct sum of $q$ copies of the spaces $E, E^{\prime}, E^{\prime \prime}$ and the maps $L, L^{\prime}$ and by applying $c^{p / q}([L])=\frac{1}{q} c^{p}([\underset{q}{[} L])$.

Finally (2.1) extends to all real $\alpha$ by continuity.

We now consider a differentiable map $f$ of a Riemannian manifold $X$ and an invariant subset $K$ and let us call $\alpha_{0}=\inf _{n} \sup _{x} \operatorname{dim} \operatorname{dil}\left[D_{x} f^{n}\right]$.

We have clearly $\alpha_{0} \geqq \sup _{x} \inf _{n} \operatorname{dim} \operatorname{dil}\left[D_{x} f^{n}\right] \geqq \operatorname{dim} \operatorname{dil} \operatorname{Sp} m$ and proving Proposition 2 is proving the converse inequality.

For all $n$ we choose $x_{n}$ such that $\operatorname{dim} \operatorname{dil}\left[D_{x_{n}} f^{n}\right] \geqq \alpha_{0}$, i.e. $c^{\alpha}\left(\left[D_{x_{n}} f^{n}\right]\right) \geqq 0$ for all $\alpha<\alpha_{0}$.

Let us fix $\alpha<\alpha_{0}, N, n>N$ and $0 \leqq j<N$. We have, by Lemma 2.1:

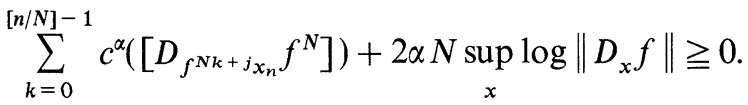

By summing over $j, 0 \leqq j<N$, we get:

$$
\begin{array}{r}
\sum_{k=0}^{n-1} c^{\alpha}\left(\left[D_{f^{k} x_{n}} f^{N}\right]\right) \geqq-3 \alpha N^{2} \sup \log \left\|D_{x} f\right\|, \text { or } \\
\int c^{\alpha}\left(\left[D_{x} f^{N}\right]\right)\left(\frac{1}{n} \sum_{k=0}^{n-1} \delta_{f^{k} x_{n}}\right)(d x) \geqq-\frac{3 \alpha N^{2} \sup _{x} \log \left\|D_{x} f\right\|}{n},
\end{array}
$$

where $\delta_{z}$ denotes the Dirac measure at the point $z$. Let $M$ be the set of vague limit 
points of the sequence of probability measures $\frac{1}{n} \sum_{k=0}^{n-1} \delta_{f^{k} x_{n}}$. All measures in $M$ are invariant and (2.2) implies $\inf \int c^{\alpha}\left(\left[D_{x} f^{N}\right]\right) m(d x) \geqq 0$. So for all $m$ in $M$ and all $N$ we get $\int c^{\alpha}\left(\frac{1}{N}\left[D_{x} f^{N}\right]\right) m(d x) \geqq 0$ and this implies, if $m=\int m_{x} m(d x)$ is the ergodic decomposition of $m$, by (1.2): $\int c^{\alpha}\left(\operatorname{Sp} m_{x}\right) m(d x) \geqq 0$, which means that there exists an ergodic measure $m_{0}$ with $c^{\alpha}\left(\operatorname{Sp} m_{0}\right) \geqq 0$, i.e. dim dil Sp $m_{0} \geqq \alpha$. This last relation being true for any $\alpha<\alpha_{0}$ proves Proposition 2 .

We also now prove Proposition 4: we consider a piecewise differentiable map of the interval and an invariant ergodic measure $m$, such that if $Q$ is the partition in critical points, we have $H(Q)<\infty$ and $\lambda=\int \log \left|f^{\prime}\right| d m>0$. For any $\delta, \frac{1}{2}>\delta>0$, we call $P_{n}^{\delta}$ the set $P_{n}^{\delta}=\left\{e^{-n \delta}<\left|f^{\prime}\right| \leqq e^{-(n-1) \delta}\right\}$ for $n$ in $\mathbb{Z}$. Remark that $P_{n}^{\delta}$ is empty when $n$ is mall enough.

The function $\log ^{+} \frac{1}{\left|f^{\prime}\right|}$ being integrable, we have

$$
\sum_{n>0} n m\left(P_{n}^{\delta}\right) \leqq \sum_{n>0} \int_{P_{n}^{\delta}} \log \frac{1}{\left|f^{\prime}\right|}<+\infty,
$$

and therefore, if we call $P \vee Q$ the partition defined by the critical points and the

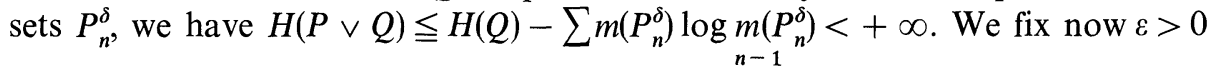
and $\chi>0$. Call $m_{n}(x)$ the measure of the atom of $\vee f^{-i}(P \vee Q)$ which contains $x$. By the ergodic theorem and (1.1) there exists $n_{0}$ so that if $n \geqq n_{0}$,

$$
\begin{array}{r}
m\left(A_{n}\right) \geqq 1-\varepsilon / 2 \text { and } m\left(B_{n}\right) \geqq 1-\varepsilon / 2, \\
A_{n}=\left\{\frac{1}{n} \log \left|\left(f^{n}\right)^{\prime}\right| \geqq \lambda(1-\chi)\right\} \\
B_{n}=\left\{-\frac{1}{n} \log m_{n} \leqq h(m)+\chi\right\} .
\end{array}
$$

where

We call $c_{n}$ the set of atoms of $\bigvee f^{-i}(P \vee Q)$ which intersects $A_{n} \cap B_{n}$. We have the following properties:

$$
\begin{aligned}
& m\left(c_{n}\right) \geqq 1-\varepsilon \\
& c_{n} \text { is made of less than } e^{n(h(m)+\chi)} \text { atoms } \\
& c_{n} \text { is an interval where } f^{n} \text { is monotone, so that the length of an } \\
& \text { atom } a \text { in } c_{n} \text { is smaller than } \\
& \left.\inf _{y \in a}\left|\left(f^{n}\right)^{\prime}(y)\right|\right)^{-1} .
\end{aligned}
$$

For any two points $y$ and $z$ in the same atom of $\bigvee_{i=0}^{n-1} f^{-i}(P \vee Q)$, we have 
$\left[\frac{\left(f^{n}\right)^{\prime}(y)}{\left(f^{n}\right)^{\prime}(z)}\right] \leqq e^{n \delta}$. The two last properties imply that the length of an atom in $c_{n}$ is smaller than $\frac{e^{n \delta}}{e^{n \lambda(1-\chi)}}$, so that we have proved that $N_{d}\left(e^{n \delta} / e^{n \lambda(1-\chi)}, \varepsilon, m\right) \leqq e^{n(h(m)+\chi)}$. Therefore

$$
\begin{aligned}
f(m) & =\lim _{\varepsilon} \varlimsup_{n} \frac{\log N_{d}\left(e^{n \delta} / e^{n \lambda(1-\chi)}, \varepsilon, m\right)}{n(\lambda(1-\chi)-\delta)} \\
& \leqq \frac{h(m)+\chi}{\lambda(1-\chi)-\delta} .
\end{aligned}
$$

We first let $\chi$ be arbitrarily small and then $\delta$ to prove $f(m) \leqq \frac{h(m)}{\lambda}$. The converse inequality comes from Proposition 1 if $f$ is differentiable, or is proved in the same way if $f$ is only piecewise differentiable and $m$ such that $H(Q)<+\infty$.

\section{Proof of Proposition 3}

The proof of proposition 3 consists in making rigorous the heuristic argument of [FKY]. We consider a differentiable map $f$ of a compact Riemannian manifold $X$, such that $D_{x} f$ satisfies a Hölder condition of order $\varepsilon$. For $x$ and $y$ close enough, let us call $\tau_{y}^{x}$ the isometry from $T_{y} X$ to $T_{x} X$ defined by parallel transport along the geodesic. The Hölder condition means that there exists $C_{0}$ and $\varepsilon>0$ such that

$$
\left\|D_{x} f-\tau_{f y}^{f x} D_{y} f \tau_{x}^{y}\right\| \leqq C_{0}(d(x, y))^{\varepsilon} .
$$

From this it follows that there exists $C_{1}$ such that, if $f^{i} x$ and $f^{i} y$ stay close enough for $0 \leqq i<n$,

$$
\left\|D_{x} f^{n}-\tau_{f^{n} y}^{f^{n} x} D_{y} f^{n} \tau_{x}^{y}\right\| \leqq C_{1}^{n}(d(x, y))^{\varepsilon} .
$$

Let $m$ be an invariant ergodic probability measure on $X$ and consider the spectrum $\operatorname{Sp} m=\left\{\lambda_{1} \geqq \ldots \geqq \lambda_{d}\right\}$ of $D_{x} f$ for $m$. If $\lambda_{1}<0$, by [R] Corollary 6.2, $m$ is carried by a finite set of points and $f(m)=0$. If $\lambda_{d} \geqq 0$, dim dil Sp $m=d$ and Proposition 3 is also true. So for proving Proposition 3 , we may choose $j$ with $\lambda_{j}<0$ and $\operatorname{dim} \operatorname{dil} \mathrm{Sp} m=j-1-\frac{\sum_{i=1}^{j-1} \lambda_{i}}{i}$. Then by Oseledets' theorem, for $m$ almost every $x$ in $X$, there exists a decreasing sequence of subspaces $E_{s_{1}}^{x}=T_{x} X \supset E_{s_{2}}^{x} \supset \ldots$ ? $E_{s_{r}}^{x} \supset E_{s_{r+1}}^{x}=\{0\}$, such that the map $x \rightarrow E_{s_{i}}^{x}$ is measurable and $\frac{1}{n} \log \left\|D_{x} f^{n} v\right\| \rightarrow \lambda_{s_{i}}$ as $n \rightarrow \infty$, uniformly on $v$ in $E_{s_{i}}^{x} \backslash E_{s_{i+1}}^{x},\|v\|=1, i=1, \ldots, r$. We now fix $\chi>0, \delta>0$. There exists $n_{0}(\delta, \chi)$ such that for any $n \geqq n_{0}, m\left(A_{n}\right) \geqq 1-\delta$ where

$$
A_{n}=\left\{x \in X,\left\|D_{x} f^{n} u\right\| \leqq(1+\chi)^{n} e^{n \lambda_{s_{k}}} \text { for all } u \text { in } E_{s_{k}},\|u\|=1 \text { and all } k\right\} .
$$

We choose now $\delta^{\prime}>0, n \geqq n_{0}$ and a set of balls $\left\{B_{i}, i \in I\right\}$ of radius $r_{n}$ such that: 


$$
\begin{gathered}
\frac{1}{2^{\varepsilon}(1+\chi)^{n}}\left\langle\frac{1}{2}, m\left(\bigcup_{I} B_{i}\right)\right\rangle 1-\delta^{\prime}, \\
r_{n}=\frac{1}{2}\left(\frac{e^{\lambda_{j}}}{(1+\chi) C_{1}}\right)^{n / \varepsilon},|I| \leqq 2 N_{d}\left(r_{n}, \delta^{\prime}, m\right) .
\end{gathered}
$$

We consider $I^{\prime} \subset I$ such that if $i \in I^{\prime}, B_{i}$ intersects $A_{n}$ in $x_{i}$ and we consider for $i \in I^{\prime}$ the ball $B_{i}^{\prime}$ of radius $2 r_{n}$ and center $x_{i}$. We have clearly $\left|I^{\prime}\right| \leqq I$ and $m\left(\bigcup_{I^{\prime}} B_{i}^{\prime}\right)>1-\delta^{\prime}-\delta$; the main step of the proof is proving Lemma 3.1:

Lemma 3.1. For $i$ in $I^{\prime}, f^{n} B_{i}^{\prime}$ can be covered by less than $K$ balls of radius $r_{n} e^{n \lambda_{j}}$,

$$
K=(1+\chi)^{n d}(2 d)^{d} \prod_{i=1}^{j} e^{n\left(\lambda_{i}-\lambda_{j}\right)} .
$$

We show now how Lemma 3.1 implies Proposition 3. The set $\bigcup_{I^{\prime}} f^{n} B_{i}^{\prime}$ can be covered by less than $K\left|I^{\prime}\right|$ balls of radius $r_{n} e^{n \lambda_{j}}$ and has a measure bigger that $1-\delta^{\prime}-\delta$. This means

$$
\begin{aligned}
N_{d}\left(r_{n} e^{n \lambda_{j}}, \delta+\delta^{\prime}, m\right) & \leqq K\left|I^{\prime}\right| \\
& \leqq 2(1+\chi)^{n d}(2 d)^{d} \prod_{i=1}^{j-1} e^{n\left(\lambda_{i}-\lambda_{j}\right)} N_{d}\left(r_{n}, \delta^{\prime}, m\right) .
\end{aligned}
$$

Remark also that $r_{n}=\frac{1}{2}(B(\chi))^{n}$, where $B(\chi)=\left(\frac{e^{\lambda_{J}}}{(1+\chi) C_{1}}\right)^{1 / \varepsilon}$. We have, by taking $\lim _{\delta \downarrow 0} \lim _{\delta^{\prime} \downarrow 0} \varlimsup_{n \rightarrow \infty} \frac{\log }{n\left(\lambda_{j}-\log B(\chi)\right)}$

$$
f(m) \leqq \frac{\log (1+\chi)^{d}+\sum_{i=1}^{j-1}\left(\lambda_{i}-\lambda_{j}\right)}{-\left(\lambda_{j}+\log B(\chi)\right)}+\frac{-\log B(\chi)}{-\left(\lambda_{j}+\log B(\chi)\right)} f(m) .
$$

Hence: $-\lambda_{j} f(m) \leqq \log (1+\chi)^{d}+\sum_{i=1}^{j-1}\left(\lambda_{i}-\lambda_{j}\right)$, and by taking $\chi$ arbitrarily small, we get $f(m) \leqq j-1-\frac{\sum_{i=1}^{j-1} \lambda_{i}}{\lambda_{j}}=\operatorname{dim} \operatorname{dil} \operatorname{Sp} m$ by our choice of $\lambda_{j}$.

Proof of Lemma 3.1: For any point $y$ in $B_{i}^{\prime}$ we consider the points $f^{n} y$ and $z$, where $z$ is such that $\exp _{f^{n} x_{i}}^{-1} z=D_{x_{i}} f^{n}\left(\exp _{x_{i}}^{-1} y\right)$, where $\exp _{v}$ denotes the exponential map from a neighbourhood of 0 in $T_{v} X$ into $X$.

By (3.1) we have

$$
\begin{aligned}
d\left(f^{n} y, z\right) & =\left\|\exp _{f^{n} x_{i}}^{-1} f^{n} y-\exp _{f^{n} x_{i}}^{-1} z\right\| \\
& \leqq C_{1}^{n}(d(x, y))^{1+\varepsilon} \\
& \leqq C_{1}^{n} r_{n} r_{n}^{\varepsilon}=\frac{C_{1}^{n} r_{n} e^{n \lambda_{j}}}{2(1+\chi)^{n} C_{1}^{n}}=\frac{r_{n} e^{n \lambda_{j}}}{2}
\end{aligned}
$$


If we consider now $C_{i}=\exp _{f^{n} x_{i}}\left[D_{x_{i}} f^{n}\left(\exp _{x_{i}}^{-1} B_{i}^{\prime}\right)\right]$, the former computation shows that for any point $f^{n} y$ in $f^{n} B_{i}^{\prime}$, there exists a point $z$ in $C_{i}$ with $d\left(f^{n} y, z\right) \leqq$ $\frac{1}{2} r_{n} e^{n \lambda_{j}}$.

From a cover of $C_{i}$ by $K$ balls of radius $\frac{1}{2} r_{n} e^{n \lambda_{j}}$, we can therefore deduce a cover of $B_{i}^{\prime}$ by $K$ balls of radius $r_{n} e^{n \lambda_{j}}$. We compute now such a $K$ : we choose in $T_{x_{i}} X$ an orthonormal basis $u_{1}, u_{2}, \ldots u_{d}$, by first choosing a basis in $E_{s r}^{x_{i}}$, then completing it into a basis in $E_{s_{r-1}}^{x_{i}}$, and so on, so that the asymptotic behaviour of $\left\|D f^{n} u_{k}\right\|$ is given by $\lambda_{d-k}$.

We have $\exp _{x_{i}}^{-1} B_{i}^{\prime} \subset\left\{v \in T_{x_{i}} X\left|v=\sum v_{k} u_{k},\right| v_{k} \mid \leqq 2 r_{n}\right\}$ and therefore

$$
\exp _{f^{n} x_{i}}^{-1} C_{i} \subset\left\{v \in T_{f^{n} x_{i}} X\left|v=\sum v_{k}\left(D_{x_{i}} f^{n}\right) u_{k},\right| v_{k} \mid \leqq 2 r_{n}\right\},
$$

where $\left(D_{x_{i}} f^{n}\right) u_{k}$ are independent vectors of $T_{f^{n} x_{i}} X$, satisfying

$$
\left\|\left(D_{x_{i}} f^{n}\right) u_{k}\right\| \leqq e^{n \lambda_{d-k}}(1+\chi)^{n} .
$$

Now cover $\exp _{f^{n} x_{i}}^{-1} C_{i}$ by parallelepipeds in $T_{f^{n} x_{i}} X$ of the following form,

$$
\left\{z\left|z-x_{0}=\sum t_{k} \frac{\left(D_{x_{t}} f^{n}\right) u_{k}}{\left\|\left(D_{x_{t}} f^{n}\right) u_{k}\right\|}\right| t_{k} \mid \leqq \frac{r_{n} e^{n \lambda_{j}}}{2 d}\right\}
$$

with less than $K$ such parallelepipeds, where

$$
K \leqq(1+\chi)^{n d}(2 d)^{d} \prod_{i=1}^{j-1} e^{n\left(\lambda_{i}-\lambda_{j}\right)} .
$$

By using the exponential map, any such parallelepiped becomes contained in some ball of radius $\frac{r_{n} e^{n \lambda_{j}}}{2}$ and this proves the lemma.

\section{Conclusions}

In this paper we introduced a number associated to a dynamical system and an invariant measure which expresses some geometrical property of the system.

For a compact metric $K$ and a map $f$, one can also define

$$
f(K)=\sup _{m \in \mathscr{E}(K, f)} f(m) .
$$

Under which conditions is the relation $\operatorname{dim} K \geqq f(K)$ true? Is there an abstract definition for $f(K)$, by cleverly mixing topological entropy and capacity (cf. Bowen $[\mathrm{Bo}]$ for instance)?

Let us come now to the comparison with numerical experiment. In [FKY], they consider some map $f$ of the square, and the computer gives a pseudo-orbit $\left\{\alpha_{n} 1 \leqq n \leqq N\right\}$. What is computed is the number $L$ of squares of radius $1 / 8$ which cover the pseudo-orbit (except some of the very first terms) and the coefficient $s=\log _{8} L$.

If one admits that the statistic of the pseudo-orbit is given by some invariant measure $m$, what is computed is actually $\log _{8} N_{d}(1 / 8, \delta(N), m)$ with $\delta(N) \rightarrow 0$ as $N \rightarrow \infty$.

If one admits also that the system has very strong self similarity properties, 
then the convergence of $\frac{\log N_{d}(\varepsilon, \delta, m)}{\log 1 / \varepsilon}$ is much faster when $\varepsilon \rightarrow 0$ than when $\delta \rightarrow 0$.

So it is sensible to believe that the number $s$ is closer to $f(m)$ than to $\varlimsup_{\varepsilon \rightarrow 0} \lim _{\delta \rightarrow 0} \frac{\log N_{d}(\varepsilon, \delta, m)}{\log 1 / \varepsilon}=c(m)$ which is the capacity of the support of the measure $m$.

It turns out that for an Axiom $A$ attractor such that the stable direction is of dimension 1, and Ruelle's measure $m$, both numbers $f(m)$ and $c(m)$ can be computed and that we have generically

$$
f(m)=\operatorname{dim} \operatorname{dil~Sp} m<c(m) .
$$

This remark is due to A. Manning and L. S. Young

A sensible reformulation fo [FKY]'s conjectures is therefore, under some conditions, a strange attractor generically admits an invariant measure satisfying $f(m)=\operatorname{dim} \operatorname{dil~Sp} m$.

Acknowledgement. I am very thankful to Michel Herman for having pointed out the inaccuracy of a previous statement of proposition 4.

\section{References}

[B] Billingsley, P. : Ergodic theory and information. New York: J. Wiley, 1965

[Bo] Bowen, R. : Topological entropy for non-compact sets. TAMS 184, 125-136(1973)

[C] Colebrook, R. : The Hausdorff dimension of certain sets of non-normal numbers. Michigan Math. J. 17, 103-116 (1970)

[D.O] Douady, A. and Oesterle, J. : Dimension de Hausdorff des attracteurs. C.R.A.S. 290, 1136-1138 (1980)

[E] Eggleston, H. G. : The fractional dimension of a set defined by decimal properties Q. J. Math. Oxford Ser. 20, 31-36 (1949)

[FKY] Frederikson, P., Kaplan, J. and Yorke, J. : The dimension of the strange attractor for a class of difference systems. Preprint

[F] Furstenberg, H. : Disjointness in ergodic theory, minimal sets and a problem in diophantine approximation. Maths. Systems theory 1, 1-49 (1967)

[K] Katok, A. : Lyapunov exponents, entropy and periodic orbits for diffeomorphisms. Publications de 1'IHES, 51, 137-174 (1980)

$\left[\mathrm{M}_{1}\right]$ Mañe, R. : On the dimension of the compact invariant sets of certain nonlinear maps. Warwick Symp. Proc. SLV in Math. (to appear)

$\left[\mathrm{M}_{2}\right]$ Mañe, R. : Lyapunov Exponents and Stable Manifold for Compact Transformations. Preprint

[O] Oseledets, V. I. : Multiplicative ergodic theorem Lyapunov characteristic numbers for dynamical systems. Trans. Moscow Math. Soc. 19, 197-221 (1968)

[R] Ruelle, D.: Ergodic theory of differentiable dynamical systems. Publication de 1'IHES, 50, 27-58 (1979)

$\left[\mathrm{R}_{2}\right]$ Ruelle, D. : Characteristic exponents and invariant manifold in Hilbert space. Preprint

Communicated by D. Ruelle

Received March 19, 1981 